\title{
Accroissement et diversification de I'offre de produits laitiers au Sénégal : la bataille industrielle du lait en poudre à Dakar et des minilaiteries à la conquête des marchés des villes secondaires
}

\author{
C. Broutin ${ }^{1} *$ V. Duteurtre ${ }^{2}$ A. Tandia ${ }^{3}$ \\ B. Touré ${ }^{3}$ M. François ${ }^{4}$
}

Mots-clés

Lait - Produit laitier - Petite entreprise - Traitement -

Diversification - Sénégal.

\section{Résumé}

Les travaux d'analyse de l'évolution de l'offre de produits laitiers au Sénégal, entre 2000 et 2005, ont mis en évidence un certain cloisonnement géographique des marchés avec une tendance à la diversification de l'offre de produits sur le marché de Dakar. Cette évolution est liée à la croissance des importations de lait en poudre et au développement d'un tissu d'industries, et de petites et moyennes entreprises / industries. La bataille commerciale que se livrent les importateurs et transformateurs industriels révèle la croissance de la demande et l'enjeu économique du marché dakarois. La filière locale, caractérisée par un accroissement du nombre de petites unités de transformation, se développe surtout dans les régions. Cependant, le dynamisme de ce secteur échappe aux décideurs politiques à Dakar du fait du confinement des minilaiteries sur les marchés des villes secondaires où elles sont implantées. L'Etat a privilégié la couverture des besoins des consommateurs dakarois avec des tarifs douaniers particulièrement bas pour la poudre de lait transformée qui bénéficie, de plus, de subventions dans les pays producteurs. Ses interventions sur la filière locale, basées sur l'intensification (insémination artificielle), $\mathrm{n}^{\prime}$ ont pas permis de lever les multiples contraintes (santé animale, alimentation, eau, gestion de la qualité, collecte du lait de brousse...). Il semble dès lors nécessaire dans l'élaboration des politiques laitières d'examiner la filière de manière plus globale et de favoriser une concertation avec l'ensemble des acteurs des deux sous-filières pour accroître l'impact des décisions politiques, notamment en termes de sécurité alimentaire et de lutte contre la pauvreté en milieu rural.
1. Gret Sénégal, s/c IRD Hann Maristes, BP 1386, Dakar, Sénégal ; Gret, campus du Jardin tropical, 45 bis avenue de la Belle Gabrielle, 94736 Nogent-sur-Marne, Cedex, France.

E-mail : broutin@gret.org

2. Gret, Dakar, Sénégal.

3. Groupes recherche action formation (Enda graf), Dakar, Sénégal.

4. Gret, Paris, France.

* Auteur pour la correspondance
INTRODUCTION : COEXISTENCE DE DEUX FILIERES

Au Sénégal, l'élevage est la deuxième activité du secteur agricole après l'agriculture : il contribue à 35 p. 100 du PIB du secteur primaire et à 4,8 p. 100 du PIB total. La production laitière nationale reste cependant très faible. Elle est estimée en 2004 à 114,2 millions de litres et ne peut répondre aux besoins nationaux en produits laitiers. La satisfaction de la demande demeure ainsi tributaire des importations de produits laitiers : elles ont représenté 250 millions d'équivalents litres de lait en 2004 (valeur de 36,7 milliards de francs CFA), soit environ le double de la production (4). Le secteur laitier est marqué depuis 1994 par deux types d'évolution apparemment contradictoires $(3,4,5)$ :

- une reprise des importations de produits laitiers, notamment la poudre de lait (environ 80 p. 100 en volume), après la baisse qui a 
suivi la dévaluation. Ceci a occasionné l'émergence et le développement d'un tissu de petites et moyennes entreprises / industries (PME/PMI) évoluant dans le reconditionnement et la transformation du lait en poudre ;

- le développement de la production laitière locale et de la transformation en zone rurale dans différentes zones agroécologiques. Ces dynamiques sont centrées sur des innovations techniques, mais également institutionnelles, pour améliorer les niveaux de production et l'accès aux marchés.

Le secteur laitier sénégalais est ainsi caractérisé par la coexistence de deux filières : une filière locale, témoin de l'implication ancienne des sociétés peules dans les échanges, et une filière d'importation de lait et de produits laitiers, témoin de la forte augmentation de la demande, liée à l'urbanisation et à l'ouverture aux marchés internationaux. Pour mieux documenter ces évolutions, une étude a été réalisée avec pour objectif d'analyser l'offre de produits laitiers issus de ces deux filières.

\section{MATERIEL ET METHODES}

L'étude sur l'évolution de l'offre de produits laitiers au Sénégal a été menée en 2005 avec l'appui des projets InfoConseil Mpea* et Paoa **. Cette étude s'est intéressée à la nature des produits, à leur origine, et à l'organisation de la mise en marché. Ce travail devait permettre d'actualiser les données de l'étude « Paysage des entreprises et environnement de la filière lait au Sénégal », réalisée en 2000 par le Gret et l'Enda graf dans le cadre du projet de recherche «MPE agroalimentaires »***. L'étude a donc permis de retracer l'évolution de l'offre en produits laitiers durant les cinq dernières années, et de faire une analyse comparative de l'offre de produits et des tendances d'évolution entre 2000 et 2005.

La méthode utilisée a reposé sur l'analyse de la bibliographie disponible, l'exploitation des diagnostics de minilaiteries financés par les projets MPE agroalimentaires et InfoConseil, qui fournissaient également des informations sur les produits et les prix des minilaiteries et des produits concurrents dans les marchés des villes secondaires, et sur une analyse de l'offre de produits (nature, prix, circuits de distribution) avec des relevés de prix dans les circuits de distribution à Dakar.

\section{RESULTATS ET DISCUSSION}

\section{Diversification de l'offre qui se poursuit}

L'analyse de l'offre de produits a permis d'identifier les différents types d'unités de transformation. La tendance à la diversification de cette offre observée en 2000 s'est confirmée avec une grande variété de produits et une grande diversité de marques ou de conditionnements pour un même produit (figure 1). Les principaux types de produits présents sur le marché sont décrits ci-après.

\section{Produits « haut de gamme » souvent importés}

Ces produits (yaourt, lait stérilisé UHT, fromages, beurre, crème fraîche, crèmes glacées) étaient vendus essentiellement à Dakar

* Projet d'accès à l'information et au conseil pour les micro- et petites entreprises agroalimentaires mené par le Gret et Enda graf sur financement du MAE et du CDE.

** Projet d'appui aux opérateurs/trices de l'agroalimentaire, exécuté conjointement par SNC-Lavalin et Cintech agroalimentaire sur financement de l'Agence canadienne pour le développement international (Acdi) et le gouvernement du Sénégal. *** Programme cofinancé par l'Union européenne dans le cadre du programme de recherche Inco, coordonné par le Gret avec les partenaires de recherche Enda graf (Sénégal), Université Cheikh Anta Diop (Sénégal), Agro PME (Cameroun), Cirad (Cameroun), AD (Guinée Bissau), Inde (Portugal), Ird (France), Gret (France). pour une clientèle haut de gamme, nationale et étrangère (expatriés et touristes). Une industrie produisait également des yaourts, du fromage blanc et de la crème fraîche à partir de lait reconstitué. L'offre de produits importés a évolué depuis 2000 avec un plus grand nombre de marques sur le marché et une augmentation des types de produits rencontrés (diversification des produits).

\section{Lait en poudre et produits dérivés de grande consommation}

On trouvait le lait en poudre sous différentes formes mais la plus courante était la vente en vrac avec un reconditionnement dans les boutiques de quartier en sachet noué (marché populaire). Il était parfois conditionné en microdose (sachet métallisé), nature ou parfumé, pour ce même marché. Le lait en boîte ou en sachet métallisé de plus grande capacité était destiné à une clientèle à revenus moyens. Le lait caillé artisanal, fabriqué avec du lait en poudre dans les quartiers, comme le lait concentré vendu dans les boutiques visaient également un marché populaire.

\section{Produits traditionnels à base de lait naturel}

Le lait caillé vendu en petites quantités à Dakar à des prix élevés était très présent dans les villes secondaires et dans les marchés ruraux à des prix très compétitifs. D'autres produits traditionnels tels que le beurre artisanal ou le diwu nior (huile de beurre) étaient également présents mais en petites quantités.

\section{Produits nouveaux fabriqués localement pour une clientèle plus large}

De nouveaux sachets de lait caillé et de yaourt liquide, fabriqués à partir de lait en poudre, ont fait leur apparition depuis quelques années à Dakar, surtout dans les libres-services et supérettes, visant plutôt une clientèle à revenus moyens. Ils étaient également distribués en microdoses, parfois glacés dans les boutiques. Les PME/ PMI qui transformaient le lait reconstitué à partir de poudre de lait ont diversifié leur offre depuis 2000 : yaourts en pot, petits-suisses, crèmes glacées, lait reconstitué pasteurisé en bouteille ou stérilisé dans des briques.

On trouvait également dans cette catégorie des produits à base de lait naturel, comme le fromage de chèvre ou plus rarement de vache, vendus dans les supermarchés et supérettes de Dakar et des zones touristiques, ou directement aux hôtels et restaurants, ainsi que du lait pasteurisé et du lait caillé, fabriqués et vendus en sachets soudés dans les villes secondaires.

\section{Circuits de distribution demeurant généralement « spécialisés »}

Les différents types de produits s'adressant à des clientèles différentes ne se trouvaient pas nécessairement dans les mêmes circuits de vente. Les circuits dépendaient également de l'origine des produits.

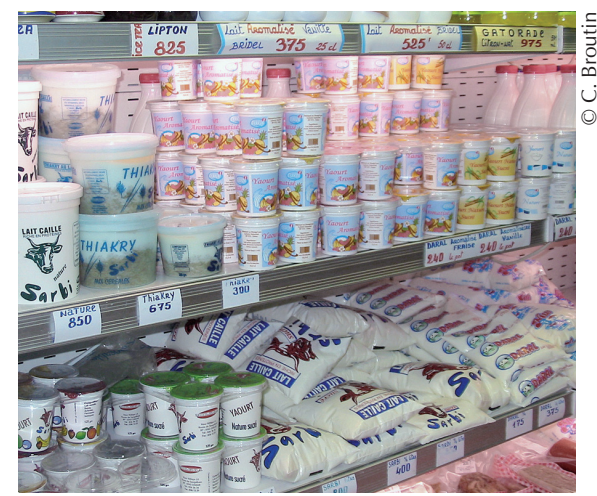

Figure 1 :

rayon produits laitiers dans une supérette à Dakar. 


\section{Circuits courts pour produits traditionnels}

Les produits traditionnels, notamment le lait caillé, étaient vendus le plus souvent dans les marchés ou à des emplacements spécifiques à Dakar (kiosques et vendeuses ambulantes). Le lait caillé était produit par les femmes d'éleveurs ou par des transformatrices qui leur achetaient le lait frais. La faible durée de conservation expliquait ces circuits courts. Il en était de même pour le lait caillé produit à partir de lait reconstitué vendu en vrac, à la louche, surtout dans les quartiers et par des hommes. La figure 2 présente les circuits de distribution des produits à base de lait cru à Dakar et dans les régions. La vente directe du producteur (effectuée généralement par sa femme) au consommateur demeurait largement majoritaire, notamment à Dakar en raison du prix rémunérateur et dans les villes secondaires où l'éleveur pouvait également vendre son lait aux petites unités de transformation.

Des intermédiaires (transformatrices ou revendeuses) dans la région de Dakar venaient collecter du lait (cru ou caillé) dans les villages, les fermes ou dans les marchés hebdomadaires proches des villages où les éleveurs apportaient le lait de leur troupeau ou celui acheté aux grandes fermes de la zone. Ces femmes le revendaient sous forme de lait cru mais plus souvent sous forme de lait caillé, dans les services de l'administration, des grandes sociétés, des banques ou dans les quartiers habités par les familles à revenus moyens et élevés (vente au porte-à-porte auprès d'un réseau de clients fidèles).

Dans les régions, la vente se faisait soit au niveau des marchés ruraux et urbains, soit aux collecteurs. Il faut noter qu'avec le développement des minilaiteries, la commercialisation s'effectuait grâce à des contrats implicites, mais aussi grâce à des contrats entre éleveurs et transformateurs (fourniture de crédits aux intrants et d'avances).

Le circuit des kiosques à Dakar lié aux fermes des Niayes constituait le troisième type de distribution, qui semblait cependant moins dynamique pour la mairie qui a décidé la destruction de certains kiosques installés sur les trottoirs (poursuite de la vente dans la rue). A Dakar, les produits qui provenaient de la ferme de Wayembam (sachets ou pots de lait caillé sucré ou non sucré) étaient présents dans les quelques supermarchés, les supérettes et libres-services et dans les stations-service. Ils étaient approvisionnés directement par la ferme. Toutefois, ce système de distribution n'absorbait qu'une faible part de la production de cette ferme. D'autres stratégies étaient utilisées, comme la vente de lait caillé sucré en pot par une camionnette ambulante qui sillonnait de manière tournante les principales artères de Dakar (place de l'Indépendance, port, marché Sandaga, etc.) (4).

\section{Circuits spécifiques, souvent courts, pour les nouveaux produits}

Les nouveaux produits (notamment les sachets de lait caillé et les yaourts) étaient présents dans les supermarchés de Dakar, mais surtout dans un nouveau réseau de distribution constitué de supérettes et libres-services implantés dans les rues passantes, généralement dans des quartiers de moyen standing ainsi que dans les stationsservice. Ils étaient approvisionnés directement par les producteurs.

La vitesse de rotation des produits était plus rapide que dans les supermarchés et les conditions de vente étaient meilleures que dans les boutiques de quartier qui ne possédaient généralement qu'un congélateur utilisé comme réfrigérateur, fréquemment ouvert le jour et éteint la nuit, et ne présentant donc pas des conditions de conservation satisfaisantes pour ces produits très périssables. Les fromages étaient vendus à Dakar dans ce même circuit.

Le réseau de distribution des produits issus des minilaiteries était constitué dans la zone sud en majorité de commerces alimentaires dont la vente de produits laitiers contribuait à la diversification des revenus. Ces boutiques étaient approvisionnées tous les jours en petites quantités. Dans la vallée du fleuve Sénégal, le réseau de distribution n'était pas encore très organisé. En dehors des ventes à l'unité sur place, les minilaiteries écoulaient les produits surtout au niveau des marchés hebdomadaires.

Même si les produits des minilaiteries commençaient à pénétrer le marché, elles auraient pu étendre leurs circuits de distribution, encore très localisés, et accroître leurs ventes par la mise en place de stratégies de marketing mettant bien en exergue les caractères
Distribution

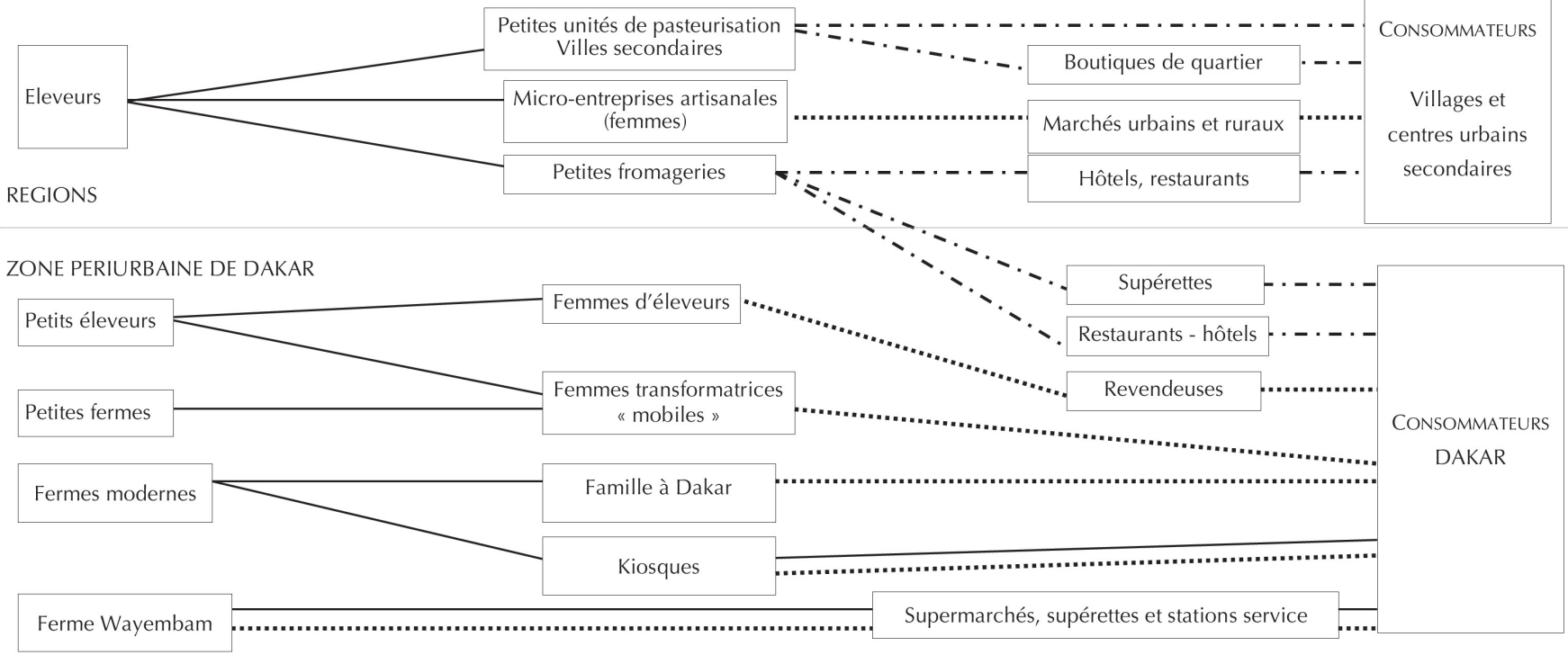


distinctifs des produits, à savoir leur origine (référence au terroir), leurs attributs à travers leur qualité nutritive et hygiénique, pour mieux informer et se rapprocher des attentes des consommateurs (4).

\section{Circuits longs pour lait en poudre et produits importés}

Le lait en poudre ainsi que les autres produits importés étaient commercialisés à travers le circuit long des importateurs, grossistes, semi-grossistes, détaillants (boutiques, supérettes...) (figure 3). Les industries (et quelques PME/PMI) qui procédaient à la reconstitution du lait en poudre pour la production de lait caillé, yaourts, etc. ou au reconditionnement (produits emballés) importaient directement le lait en poudre et les produits empruntaient ensuite le même circuit.

Les principaux importateurs de poudre de lait étaient Nestlé (marque Nido), Satrec (marques Vitalait, Roilait, Best lait...), Senelac, Senico (marques Halib Sunulait et Bonlait), Socidig, Sonia, Sosepal (marque Baralait), Ucodis (marque Laicran). Certains importateurs réexportaient le lait vers le Mali, la Gambie et la Guinée. Moins de 10 p. 100 des importations de lait en poudre subissaient une transformation industrielle, le reste était transformé par les PME, les unités artisanales et vendu au détail. Les principaux importateurs de produits laitiers étaient Sofiex, Patisen et Spca qui approvisionnaient l'ensemble du pays et représentaient les grandes marques du secteur telles que Président, Elle \& Vire, Bridel, etc. (7).

Les principaux clients des importateurs étaient les grandes surfaces, les grossistes et semi-grossistes, les hôtels et restaurants, quelques transformateurs et détaillants. La majorité des grossistes qui alimentaient Dakar, sa banlieue et les autres régions du Sénégal en produits laitiers et autres produits alimentaires (riz, huile, détergents...) étaient installés au centre ville. Les grossistes vendaient à des semi-grossistes installés dans les marchés de Dakar et dans les régions, à des détaillants et des transformateurs. Environ la moitié des ventes de lait en poudre concernait Dakar, l'autre moitié partait dans les régions. Les détaillants reconditionnaient le lait en petits sachets noués.

\section{Croissance de l'offre et conditionnement des produits}

\section{Croissance de l'offre de lait en poudre}

Les importations de produits laitiers ont augmenté régulièrement jusqu'en 1993 où elles ont atteint 223 millions d'équivalents litres de lait, dont 93 p. 100 sous forme de lait en poudre. Elles ont chuté en 1994 et en 1995, suite au changement de parité du franc CFA qui a fait doubler les prix à la consommation sans effet durable sur la consommation. En effet, les importations ont été depuis en augmentation régulière avec, en 2000, 237 millions d'équivalents litres importés pour une valeur de 27 milliards de Fcfa. En 2004, les importations de produits laitiers ont représenté 34794 t, soit l'équivalent de 250 millions de litres de lait, pour une valeur de 36,7 milliards de Fcfa. Le lait en poudre a représenté 88 p. $100 \mathrm{du}$ tonnage (6). Il était importé dans des sacs mais également, pour un faible volume, en sachet ou pot métallique (importations notamment de produits Nestlé).

\section{Activités de reconditionnement florissantes}

Les entreprises qui reconditionnaient le lait en poudre importé étaient plus nombreuses. En 2000, la société Satrec était seule sur ce marché. En 2005, six entreprises concurrentes se sont développées : Sosepal, Senico, les établissements Meroueh, Senelac, les Mamelles Jaboot (qui produisaient également du yaourt) et Promasidor (Cowbell) qui a stoppé ses activités en 2005. La société Satrec a développé son offre depuis 2000, notamment en sachet microdose avec la création de nouvelles marques : Roilait, Bestlait et Vitadiet, présenté comme un produit diététique (figure 4).

L'histoire de Cowbell reflète l'enjeu de ce marché et l'âpre concurrence entre les entreprises. Cowbell faisait partie du groupe Promasidor (société d'une famille anglaise basée en Afrique du Sud) qui a démarré ses activités il y a plus de 20 ans dans l'ex-Zä̈re (actuelle République démocratique du Congo) en commercialisant de petites doses de lait en poudre en sachets de 22,5 g et qui est présent dans plus de 24 pays d'Afrique. Début 2000, Cowbell s'est installé au Sénégal et a démarré en 2002 une usine de production et d'ensachage représentant un investissement d'environ 2 milliards de Fcfa

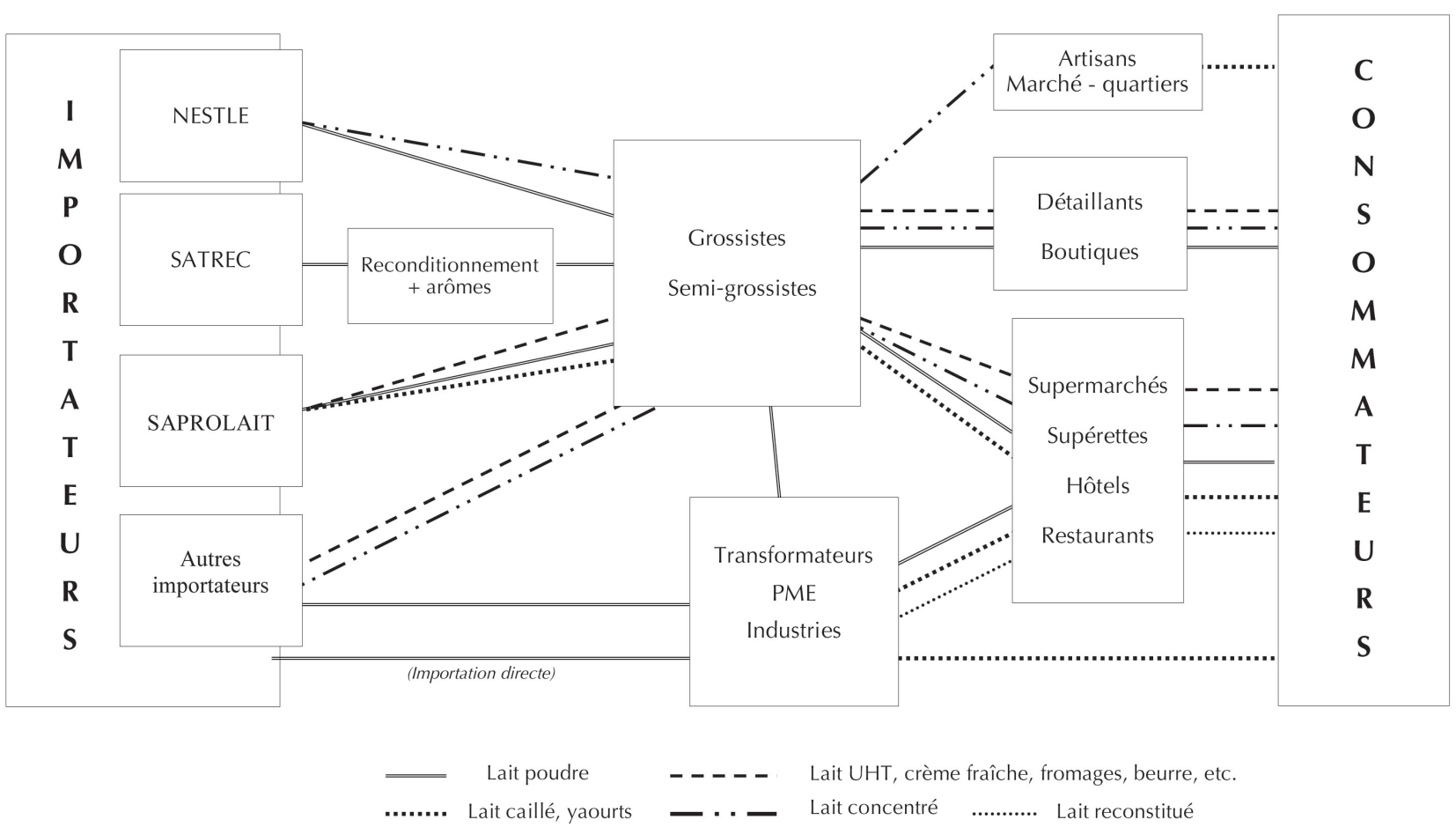

Figure 3 : circuits des produits importés. 
et employant 120 personnes. Les objectifs de la société étaient non seulement de s'implanter en Afrique de l'Ouest avec comme rampe de lancement le Sénégal, mais aussi de concurrencer la Satrec, dont les emballages Vitalait étaient pratiquement identiques à ceux de Cowbell. La Satrec décida d'ester en justice contre Cowbell, au vu de la ressemblance quasi parfaite entre l'emballage des produits (format, couleur et verre de lait sur le sachet). Le tribunal a confirmé la contrefaçon et Cowbell a été interdit de vente en 2003. Cette société a été mise en faillite et placée en liquidation le $1^{\mathrm{er}}$ avril 2005 (8).

\section{Variations de prix très inégales}

D'après les relevés effectués sur les lieux de distribution, les prix du lait en poudre emballé ont augmenté entre 20 (conditionnement en sachet microdose) et 35 p. 100 (vente en pot métallique) entre 2000 et 2005 ; celui du lait en poudre en vrac n'a gagné que 10 p. 100 dans le même temps. On trouvait même dans certaines boutiques des sachets noués de $500 \mathrm{~g}$ au même prix qu'en 2000 (900 Fcfa). Il y aurait eu davantage d'importations de lait en poudre écrémé ou avec addition de matières grasses végétales (voire dé-protéiné), permettant de mettre sur le marché un produit à plus bas prix (mais de moindre qualité nutritionnelle), qui pouvait expliquer la stagnation des prix du lait en poudre vendu en vrac dans les boutiques, mais les statistiques n'ont pas permis de confirmer cela et la vente en vrac ne fournissait aucune information au consommateur sur le produit.

\section{Industries et PMI de transformation du lait en poudre ont diversifié leur offre}

\section{Nouvelles entreprises à Dakar}

Entre 2000 et 2005, seule une PME semble avoir stoppé ses activités et Nestlé a cessé sa production de lait concentré pour orienter ses activités vers l'importation et la distribution de lait concentré et de poudre de lait (7). Le secteur est marqué en 2005 par l'arrivée de trois nouvelles entreprises [La laiterie dakaroise, Le Taif et la Société industrielle agroalimentaire (Siaa)], toutes implantées dans la région de Dakar, qui ont investi ce marché en plus des quatre entreprises déjà présentes en 2000 (Saprolait, les Mamelles Jaboot, SIM et Milkoa). Hormis Saprolait, première industrie laitière implantée au Sénégal, toutes ces entreprises avaient moins de dix ans. Elles produisaient et distribuaient essentiellement du lait caillé sucré et non sucré (en sachets et en pots) et des yaourts (natures, aromatisés, aux fruits, etc.) en pots.

Saprolait produisait également du fromage blanc de campagne et de la crème fraîche, tandis que les Mamelles Jaboot et Le Taîf s'étaient spécialisés dans la production de cakry (mélange de lait caillé et de granules de farine de mil, issues du roulage de la farine humidifiée puis tamisée). Les Mamelles Jaboot fabriquaient également du fromage fondu (en barquette en plastique de $200 \mathrm{~g}$ ) et conditionnaient du lait en poudre (sachets métallisés de 22,5 et 500 g). SIM fournissait des crèmes glacées en bacs ou en pots. Siaa a investi 2,5 milliards de Fcfa en 2005 pour développer son activité de production de lait stérilisé UHT Candia entier et demi-écrémé (à partir de poudre de lait importée, cahier des charges Candia), vendu en briques d'un litre (contrat de licence Tetra Pak), et souhaitait développer toute la gamme Candia : lait écrémé et aromatisé, mélange de jus et de lait. La laiterie dakaroise produisait du lait pasteurisé entier et demi-écrémé Daral (bouteilles d'un demilitre et d'un litre) et du lait concentré sucré Laicran $(2,4,7)$.

Nestlé, implantée depuis 1961 à Dakar, exploitait depuis 1973 une unité de fabrication de lait concentré sucré et non sucré à partir de poudre de lait importée et d'huile de beurre. Le lait frais local collecté au niveau des élevages traditionnels de la région de Dahra (partenariat Nestlé - producteurs locaux) était mélangé au lait en poudre. La société a stoppé sa production de lait concentré à la fin de l'année 2002 à la suite de sa restructuration : la production a été délocalisée vers le Ghana et Nestlé Sénégal importe et distribue les produits vendus sous la marque Nestlé (et non plus Gloria). Elle importe et distribue également le lait en poudre Nido. Les centres de collecte de lait (dix), représentant une valeur d'environ 200 millions de Fcfa, ont été rétrocédés à l'Etat sénégalais par Nestlé qui, à travers le Projet d'appui à l'élevage (Papel), en a confié la gestion à une organisation d'éleveurs, l'Union des producteurs et des préposés au rayon laitier (Uppral) (7).

\section{Développement de produits pour concurrencer les importations}

On observe en particulier un accroissement de l'offre de yaourt en pot (figure 5) et de lait stérilisé et UHT à base de lait en poudre. En 2000, seule la société Saprolait produisait du yaourt en pot ; les Mamelles Jaboot fabriquaient déjà du yaourt liquide vendu en sachet, alors que toutes les autres entreprises ne produisaient que du lait caillé. En 2005, quatre autres sociétés fabriquaient du yaourt en pot. Le produit était vendu à des restaurants et hôtels, et dans les supermarchés et supérettes, mais également dans les boutiques de quartier à des prix très variables. En 2000, le pot de yaourt, nature ou sucré, était vendu dans les supermarchés entre 210 et $270 \mathrm{Fcfa} /$ pot et entre 325 à $375 \mathrm{Fcfa} /$ pot dans les boutiques de quartier (soit 40 à 50 p. 100 plus cher). En 2005, le pot de yaourt nature fabriqué localement était vendu dans les supermarchés et supérettes en moyenne à $235 \mathrm{Fcfa} /$ pot, alors que le pot de yaourt importé le moins cher était vendu à plus de $400 \mathrm{Fcfa} /$ pot. Il y avait donc une tendance à la baisse des prix des produits locaux, sans doute liée à la concurrence, même si le yaourt restait un produit cher. Ce développement de l'offre s'est traduit effectivement par une baisse des importations : 1900 t en 1994 et seulement $170 \mathrm{t}$ en 2002 pour une valeur qui est passée de 3960 millions à 209 millions de Fcfa (d'après la Direction de la prévision et des statistiques). La production de yaourt est donc une activité d'import-substitution réussie.
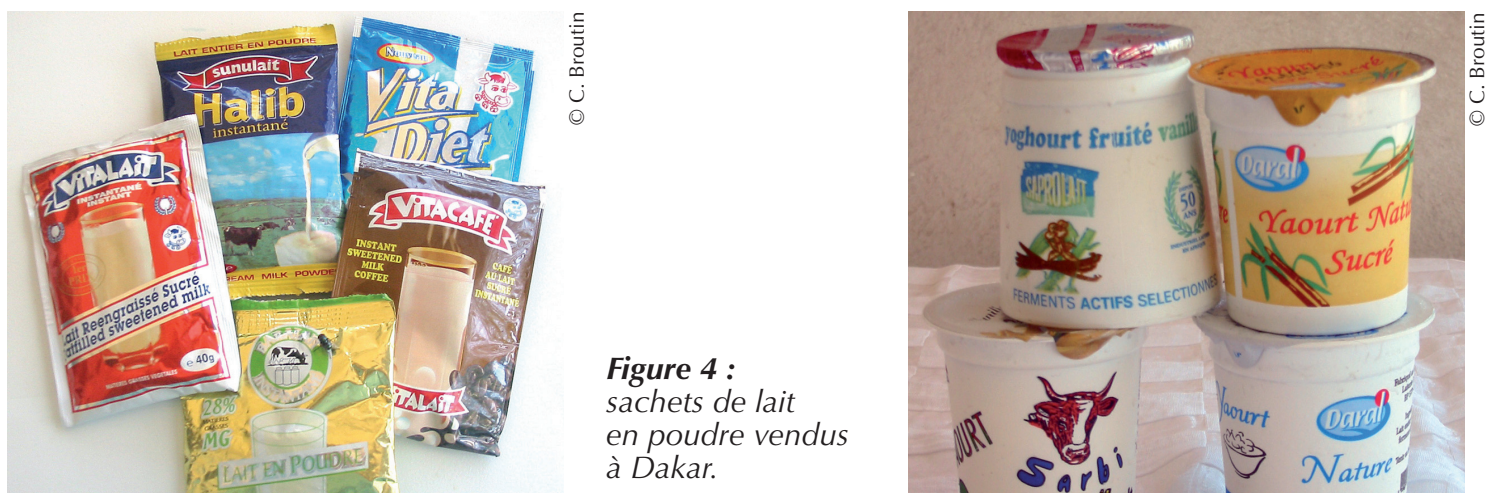

Figure 5 :

yaourts à base de lait en poudre fabriqués à Dakar. 
Les chiffres des importations mettent en évidence une forte hausse du lait UHT, dont le volume est passé de presque 4500 t en 1995 à plus de $9300 \mathrm{t}$ en 2002. Ceci explique sans doute que des industries se soient positionnées sur ce type de produit avec la mise en marché récente d'un lait reconstitué pasteurisé par La laiterie dakaroise sous la marque Daral et d'un lait reconstitué UHT produit par Siaa sous la marque Candia (licence). Le prix de ces produits « locaux » est très compétitif, puisqu'en 2005 le litre de lait UHT importé a varié entre 780 et $1050 \mathrm{Fcfa}$, le litre de lait reconstitué pasteurisé Daral entre 600 et 650 Fcfa, et le litre de lait reconstitué UHT Candia entre 500 et 650 Fcfa.

\section{Développement de nouveaux produits pour élargir et diversifier la gamme}

Les Mamelles Jaboot et Le Taif (pour un volume plus faible) ont mis sur le marché du cakry. D'après le promoteur d'une de ces entreprises, la demande en cakry est très forte et son produit bien apprécié. Face à l'évolution de l'offre en produits laitiers au Sénégal, l'entreprise compte se diversifier en associant le lait à différents types de produits.

\section{Nécessité de veiller à une bonne information du consommateur sur les produits obtenus à partir de poudre de lait}

L'analyse des produits présents dans les circuits de distribution de Dakar a montré que l'information des consommateurs n'était pas toujours satisfaisante, notamment au niveau de l'étiquetage mais également dans les messages publicitaires. Par exemple, l'utilisation de lait en poudre (et sa nature) pour la confection de yaourt et de lait caillé n'était pas mentionnée (indications données : « lait entier pasteurisé », " lait enrichi ») alors que la réglementation l'exigeait (indication de la nature et de la quantité de lait en poudre ajouté si cette addition est supérieure à $5 \mathrm{~g}$ de poudre pour $100 \mathrm{~g}$ de lait). Sur certains sachets figuraient une femme peuhle et sa calebasse, une vache locale, un acacia, symboles de la production locale. Le lait pasteurisé produit au Sénégal était présenté dans les magasins dans les rayons réfrigérés avec la mention « lait frais pasteurisé ». De nombreux gérants de magasin rencontrés pensaient ainsi que le produit était fabriqué à partir de lait cru local.

Le lait UHT « local » était présenté sous la marque Candia grand lait (présentation identique au produit Candia européen) (figure 6). La mention « lait UHT reconstitué » apparaissait en tout petit sur le côté de la brique. Il se positionnait en concurrent direct des laits

UHT importés (Bridel, Président, etc.) en jouant sur un emballage et un conditionnement qui pouvaient faire croire au consommateur qu'il achetait du vrai lait Candia importé. La publicité du produit dans les différents médias mettait cependant plutôt en avant le caractère « local » du produit, telle la campagne médiatique de

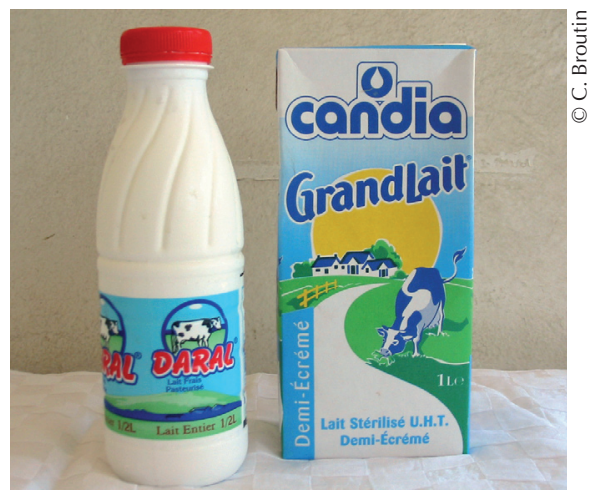

Figure 6 :

lait pasteurisé et UHT à base de lait en poudre vendu à Dakar. lancement qui indiquait : «première unité de lait pasteurisé UHT d'Afrique », " un lait made in Sénégal, reconnu et labellisé par la plus grande marque de lait européenne », " le lait économique Candia, étant produit localement, sera proposé au consommateur à un prix plus abordable que le lait importé ».

A signaler également la mise sur le marché de lait en poudre reconditionné (marque Vitadiet *), présenté comme un produit diététique (qui renvoie à une alimentation saine, à une contribution à une meilleure santé, à la prévention de risques de maladies), sans qu'il ait été clairement indiqué sur le sachet les vertus diététiques du produit : indications sur le devant du sachet « lait en poudre à matières grasses végétales » (sans mentionner qu'il était sucré avec des édulcorants chimiques); au dos « lait en poudre à MGV, riches en vitamines $\mathrm{A}$ et $\mathrm{D}$ » et composition « lait en poudre, lait à MGV édulcoré à l'aspartame et à l'acésulfame $\mathrm{K}$, vitamines $\mathrm{A}$ et $\mathrm{D}$ », produits que sans doute peu de consommateurs connaissaient.

\section{Peu de produits emballés à base de lait frais sur le marché dakarois mais présence croissante sur les marchés des villes secondaires}

\section{Offre faible à Dakar malgré une demande des consommateurs}

La ville de Dakar, qui constituait le marché de consommation des produits laitiers le plus important, était relativement mal approvisionnée en produits laitiers issus de la production locale. On trouvait dans les marchés essentiellement l'huile de beurre, produit traditionnel obtenu à partir de la crème extraite après caillage. Les consommateurs s'approvisionnaient également auprès des vendeuses de lait caillé présentes dans quelques marchés mais qui vendaient surtout au porte-à-porte à un réseau de clients fidélisés («abonnés »). Le lait provenait des fermes autour de Dakar mais également des élevages traditionnels des Niayes et de la petite côte (zone de Mbour à $80 \mathrm{~km}$ de Dakar). Le prix de ces produits vendus généralement en vrac étaient également assez élevés : 600 à $700 \mathrm{Fcfa} / \mathrm{L}$, sachant que les fermes intensives de Dakar le vendaient à $400 \mathrm{Fcfa} / \mathrm{L}$. La production de ces fermes était estimée entre 6000 et $7000 \mathrm{~L}$ mais elles n'avaient pas réellement développé de circuits de vente des produits, malgré des investissements réalisés après 2000 pour Wayembam afin de proposer du lait pasteurisé et des produits emballés (6). Les enquêtes réalisées en 2002 auprès de 400 ménages consommateurs de lait caillé (1) avaient pourtant mis en évidence un nombre relativement important de consommateurs de ces produits et leur souhait d'augmenter leur consommation.

\section{Essor des produits des minilaiteries dans les régions, le plus souvent autour des villes secondaires}

A côté de la vente de lait caillé traditionnel par les femmes d'éleveurs, notamment pendant l'hivernage, de petites entreprises de transformation se sont développées : une quarantaine a été identifiée en 2005 contre une dizaine en 2000. Celles-ci ont généralement bénéficié d'appuis de projet ou de structures. Elles étaient pratiquement toutes implantées dans des villes secondaires, dans des zones de production (figure 7).

Ces unités se caractérisaient par un aménagement du lieu de production et des volumes transformés plus importants, mêmes s'ils demeuraient modestes (20 à 400 L/jour et jusqu'à $700 \mathrm{~L} /$ jour). Le niveau d'équipement était faible (marmites en inox, réchauds

\footnotetext{
* Marque de produit présent sur le marché européen à base d'algues marines, ayant la réputation d'augmenter le métabolisme des graisses et des sucres, parce que l'iode qu'elles contiennent aurait un effet sur la glande thyroïde, et ainsi favoriser une perte de poids.
} 


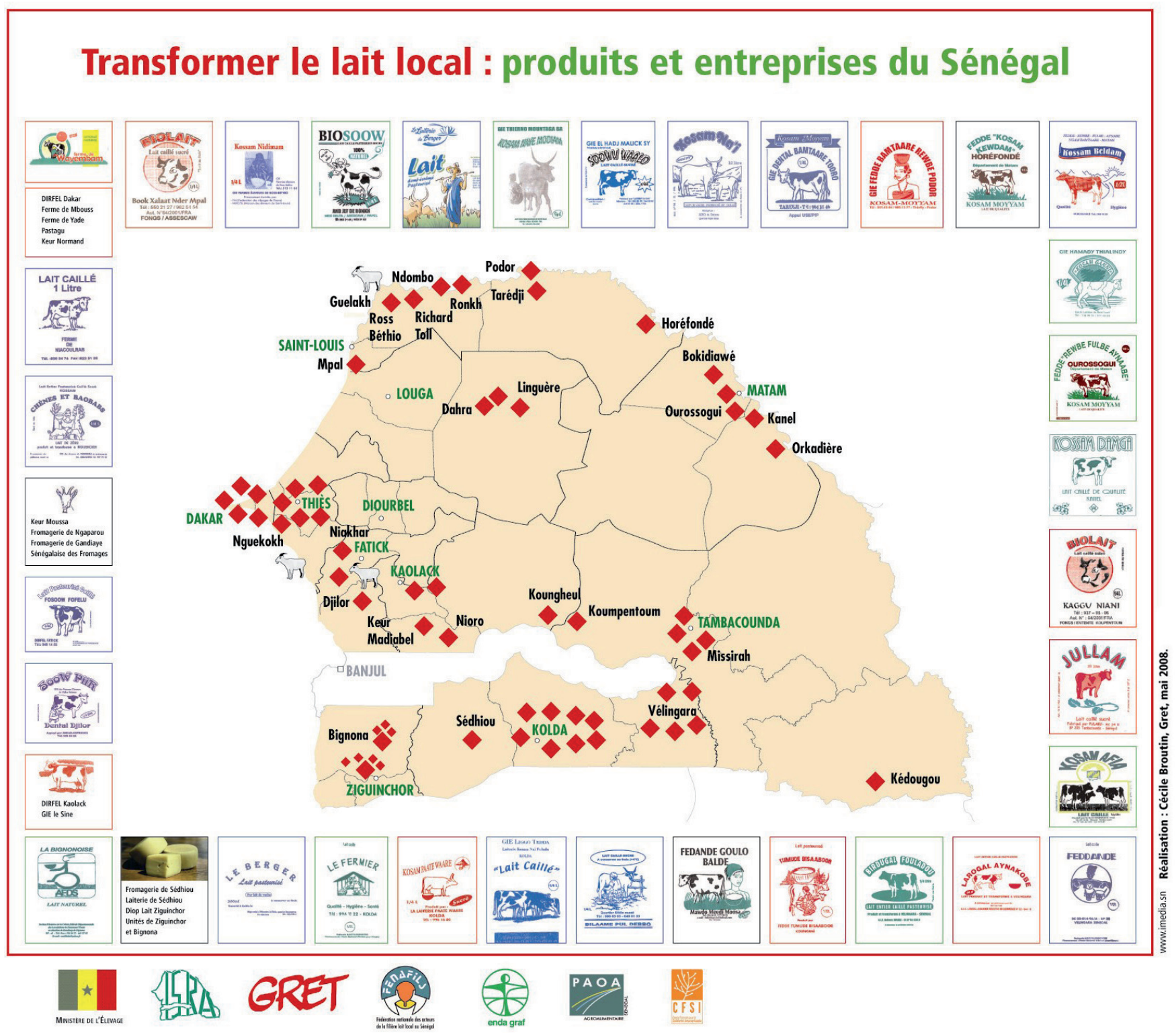

Figure 7 : localisation des petites entreprises de pasteurisation ou minilaiteries.

à gaz, soudeuse sachets, réfrigérateur et glacière). La production demeurait encore relativement faible et irrégulière (cessation temporaire d'activités pendant la période sèche ou utilisation du lait en poudre).

Les minilaiteries fabriquaient du lait caillé sucré et non sucré ainsi que du lait pasteurisé, conditionné en sachet imprimé (nom de marques, dessins...) qui donnait de la valeur au produit, lui conférait une image de qualité et était apprécié des consommateurs (1). En revanche, l'origine locale de la matière première n'était pas toujours bien mise en valeur (nom de marque, identification des produits, vaches de race Holstein sur le sachet).

Ces unités s'approvisionnaient auprès d'éleveurs situés dans un rayon de 20 à $30 \mathrm{~km}$ (collecte le plus souvent à vélo). Les produits étaient vendus à l'unité ainsi que dans les boutiques de quartier et supérettes dans certaines villes plus importantes. Rares étaient celles qui distribuaient en dehors de la ville, notamment en raison de l'absence de moyen de distribution réfrigéré. Ces produits se situaient donc sur des marchés géographiquement différents des produits emballés à base de lait en poudre qui étaient peu commercialisés dans les villes secondaires ou à des prix plus élevés.
Les produits des minilaiteries étaient surtout concurrencés par la vente de lait caillé en vrac par les femmes d'éleveurs pendant une partie de l'année (hivernage) et par la transformation artisanale de lait en poudre qui se développait dans les villes secondaires. Cependant, le lait local restait compétitif sur ces marchés. Il était en effet acheté par les minilaiteries entre 200 et $250 \mathrm{Fcfa} / \mathrm{L}$ alors que le coût du litre de lait reconstitué se situait autour de 200 à $225 \mathrm{Fcfa}$.

Les transformateurs étaient cependant confrontés à des problèmes d'approvisionnement pendant la saison sèche où l'offre était faible et où la production nécessitait l'utilisation de compléments alimentaires (tourteau d'arachide, de sésame, graine de coton) alors que l'accès n'était pas toujours facile. Les coûts de la collecte réfrigérée élevés et la faible capacité d'investissement de ces entreprises ne permettaient pas de collecter le lait à plus d'une trentaine de kilomètres. Le recours à la lactoperoxydase (substance bactériostatique pour les lactobacilles et les streptocoques, bactéricide pour le genre Pseudomonas et pour Escherichia coli) permettrait d'envisager la collecte de lait de brousse, mais son utilisation n'était pas officiellement autorisée au Sénégal. 


\section{CONCLUSION}

\section{Bataille du lait en poudre à Dakar : meilleur accès aux produits laitiers pour les consommateurs, forte concurrence sur le marché dakarois et importants budgets publicitaires}

Le développement de l'offre de produits (lait reconditionné et produits transformés) traduisait à la fois la forte demande en produits laitiers à Dakar mais également dans les villes secondaires et la rentabilité de ces activités. Les budgets publicitaires des industries et PMI étaient révélateurs de l'enjeu économique du secteur. Ces sociétés étaient ainsi très présentes sur les panneaux publicitaires de toutes les grandes artères de la ville, aussi bien pour les marques de lait reconstitué que pour les marques de produits importés (Bridel, notamment). D'après un article publié dans le journal Performances d'octobre 2005, le montant des publicités sur les deux chaînes de télévision nationales a atteint, au mois d'août 2004, 88 millions de Fcfa. Le secteur laitier se plaçait ainsi au premier rang des investissements publicitaires au Sénégal. La transformation locale du lait en poudre commençait également à concerner les produits importés, notamment le yaourt et surtout le lait UHT, sur un marché où des marques européennes étaient bien implantées.

Il faut souligner l'impact positif du développement de l'offre qui avait tendance à faire chuter les prix et qui permettait de mettre à disposition des consommateurs des produits conditionnés adaptés (microdoses), même si les produits emballés étaient encore trop chers pour une partie de la population dakaroise et inaccessibles pour la majorité des autres sénégalais. Ces produits étaient de meilleure qualité sanitaire que les produits conditionnés en sachets noués et que la vente en vrac de lait caillé, mais sans doute de moins bonne qualité nutritionnelle que les produits à base de lait cru. Cependant, il est nécessaire de veiller à une meilleure information des consommateurs sur l'origine, la qualité et la composition nutritionnelle des produits, et à ce que l'Etat assure une concurrence loyale. De plus, le développement de la filière locale présente d'autres enjeux en termes notamment de lutte contre la pauvreté rurale.

\section{Développement des minilaiteries dans les régions qui pourraient étendre leurs circuits de distribution et envisager de se positionner sur le marché dakarois}

Pendant que les industries et PMI de reconditionnement et de transformation du lait en poudre importé se «bagarraient » sur le marché de Dakar, des minilaiteries se multipliaient et faisaient leurs premières armes sur les marchés urbains des villes secondaires. En cinq ans, le nombre de minilaiteries a été multiplié par quatre, montrant le dynamisme de ce secteur. La mise en œuvre de bonnes pratiques d'hygiène à travers un guide, validé en novembre 2005 par les organisations professionnelles et l'Etat, pourrait contribuer à améliorer la qualité et assurer l'innocuité des produits. Les produits présentaient des avantages comparatifs et avaient d'importants atouts : le prix des produits était en effet plus faible et pourrait, malgré le coût du transport réfrigéré, concurrencer les produits industriels, notamment sur le marché dakarois. Ils bénéficiaient également d'une image positive liée à l'origine de la matière première (référence au terroir) et à leurs attributs nutritionnels.

Malgré les appuis fournis au développement de la production locale, ceux-ci demeuraient insuffisants pour parvenir à mieux approvisionner les minilaiteries, notamment pendant la saison sèche. L'environnement réglementaire et les politiques publiques ne favorisaient pas non plus leur développement, contrairement aux industries de transformation du lait en poudre qui bénéficiaient d'un prix subventionné sur le marché international et un faible taux de taxation douanière de leur matière première, d'un faible suivi de l'application de la réglementation (étiquetage, publicité). Il y a donc matière à réfléchir sur des mécanismes et dispositifs qui à la fois permettent au plus grand nombre de consommateurs d'accéder à des produits laitiers et à la filière locale de se développer et de reconquérir une partie du marché afin de développer l'emploi et les revenus, notamment des éleveurs.

\section{Nécessité de définir des politiques laitières qui prennent davantage en compte le développement des filières locales}

L'étude de l'offre de produits laitiers au Sénégal met en évidence la grande diversification du marché avec une grande variété de produits laitiers importés ou locaux et de qualité qui sont diversement valorisés sur le marché sénégalais. On note une dynamique d'industrialisation du secteur et une augmentation des investissements privés, y compris dans la valorisation de la production locale. L'étude montre également un certain cloisonnement physique ou géographique des marchés lié aux coûts de transport et à l'organisation des marchés et de la filière locale qui se développe surtout dans les régions, expliquant que la production locale et les produits transformés soient partiellement «protégés » de la concurrence des importations et des produits industriels à base de lait en poudre (les produits à base de lait cru sont commercialisés essentiellement dans les villes secondaires où les produits à base de poudre de lait sont relativement peu présents). Ce confinement sur des marchés secondaires les rend cependant peu visibles. Ces minilaiteries se sont développées souvent avec l'appui de projet et d'institutions mais sans véritable soutien de l'Etat alors que ces entreprises jouent un rôle déterminant dans la connexion entre production locale périurbaine et rurale, et marchés de consommation. L'Etat a privilégié la couverture des besoins des consommateurs urbains, notamment à Dakar, avec des tarifs douaniers particulièrement bas pour la poudre de lait transformée par les industries (taxe de 5 p. 100 à l'importation), poudre qui bénéficie, de plus, de subventions à la production et à l'exportation dans les pays producteurs. Depuis ces travaux, les prix sur le marché international ont beaucoup augmenté, mais l'Etat a décidé d'exonérer de toutes taxes (taxes douanières et TVA) les importations de lait en poudre afin de limiter la hausse des prix aux consommateurs.

Les interventions de l'Etat sur la filière locale ont surtout concerné l'appui à l'intensification (notamment par des programmes d'insémination artificielle), mais sans choix précis des zones à privilégier et sans concertation avec les minilaiteries qui seraient en mesure de valoriser l'accroissement attendu de la production. Les éleveurs qui approvisionnent ces unités semblent avoir assez peu bénéficié des soutiens de l'Etat et soulignent surtout d'autres contraintes importantes à lever (santé animale, alimentation, eau). Il en est de même pour les petites unités de transformation et les éleveurs plus éloignés des centres urbains qui réclament des appuis de l'Etat. Finalement, les grands projets de l'Etat ne semblent pas avoir eu d'impact significatif sur la filière lait locale, alors que de nombreux problèmes nécessitent d'être réglés (conditions de collecte du lait de brousse, gestion de la qualité, santé animale, alimentation des troupeaux, eau, etc.). Il semble dès lors nécessaire dans l'élaboration des politiques laitières d'examiner la filière de manière plus globale et surtout de favoriser une concertation avec l'ensemble des acteurs des deux sous-filières pour accroître l'impact des décisions politiques en termes de sécurité alimentaire et de lutte contre la pauvreté, qui touchent davantage le milieu rural. 


\section{BIBLIOGRAPHIE}

1. BROUTIN C., SOKONA K., NDOYE F., MARPEAU G., FRANÇOIS F., 2002. Marché et consommation des produits laitiers à Kolda, Gret, Enda graf. In : Atelier de restitution MPE agroalimentaires, Gret, Enda graf, Dakar, Sénégal, oct. 2002. www.gret.org/incompe

2. BROUTIN C., SOKONA K., TANDIA A., 2000. Paysage des entreprises et environnement de la filière lait au Sénégal. Dakar, Sénégal, Gret / Enda graf, 57 p. www.gret.org/incompe

3. DIEYE P.N., BA DIAO M., BROUTIN C., DUTEURTRE G., DIA D., DIA SOW F., 2005. La filière lait et produits laitiers. In : Etat des filières agricoles au Sénégal (vers. 28.06.2005). Dakar, Sénégal, Isra-Bame, p. 144-157.

4. DIEYE P.N., BROUTIN C., BA DIAO M., DUTEURTRE G., LY C., 2005. Synthèse bibliographique : filières lait et produits laitiers au Sénégal. Dakar, Sénégal, Réseau de recherches et d'échanges sur les politiques laitières, $40 \mathrm{p}$.
5. DUTEURTRE G., DIEYE P.N., DIA D., 2005. L'impact des importations de volailles et de produits laitiers sur la production locale au Sénégal. In : Ouverture des frontières et développement agricole dans les pays de I'Uemoa. Dakar, Sénégal, Isra-Bame, 78 p. (Etudes et documents vol. 8, $\left.\mathrm{n}^{\circ} 1\right)$

6. INFOCONSEIL, PAOA, 2006. Etat des lieux de la filière lait et produits laitiers au Sénégal. Dakar, Sénégal, Gret / Enda graf, 94 p. www. infoconseil.sn

7. MISSION ECONOMIQUE, 2004. Les produits laitiers au Sénégal. Fiche de synthèse. Dakar, Sénégal, ambassade de France, 4 p. http:// Www.missioneco.org/senegal/

8. PERFORMANCE MANAGEMENT, 2005. Publicité télévisée, oct. 2005 388000000 F dépensés au mois d'août. Dakar, Sénégal, RTS / 2sTV, $\mathrm{n}^{\circ} 25$, p. 21.

Accepté le 01.10.2009

\section{Summary}

Broutin C., Duteurtre V., Tandia A., Touré B., François M. Increase and Diversification of Dairy Product Supplies in Senegal: Industrial Fight of Dried Milk in Dakar and Small Dairy Units' Effort to Conquer Secondary Cities' Markets

Studies conducted in Senegal between 2000 and 2005 showed that markets for dairy products were geographically segregated and that the supply of dairy products in Dakar was increasingly diversified. This evolution was related to an increase in the import of dried milk, and to the development of an industrial make-up and of small/medium-sized enterprises and industries. The competition between dairy trading and processing companies revealed an increase in the demand and Dakar market's economic challenge. The local dairy subsector, characterized by an increase in the number of small processing units, was mainly expanding in rural areas. But political decision makers in Dakar were not involved in the dynamics of this local subsector because small dairies were remote, being confined to secondary cities. National policies focused on covering Dakar consumers' needs with imports at particularly low customs rates for processed dried milk, which was, moreover, subsidized in industrialized countries. State intervention in the local subsector, based on promoting intensification via artificial insemination, did not lead to the elimination of the many constraints (animal health, feed, water, quality control, milk collection...). It seems therefore necessary to develop new dairy policies that would consider the sector globally and promote a dialog between all parties involved in the two subsectors to reach political objectives, especially with regard to food safety and poverty eradication in rural areas.

Keywords: Milk - Milk product - Small enterprise Processing - Diversification - Senegal.

\section{Resumen}

Broutin C., Duteurtre V., Tandia A., Touré B., François M. Crecimiento y diversificación de la oferta de los productos lecheros en Senegal: la batalla industrial de la leche en polvo en Dakar y de las mini lecherías a la conquista de mercados de las ciudades secundarias

Los estudios de análisis sobre la evolución de la oferta de los productos lecheros en Senegal, entre 2000 y 2005, mostraron una cierta división geográfica de los mercados, con una tendencia a la diversificación de la oferta de los productos en el mercado de Dakar. Esta evolución está relacionada con el crecimiento de las importaciones de la leche en polvo y el desarrollo de una red de industrias y de pequeñas y medias empresas / industrias. La batalla comercial que se lleva a cabo entre importadores y transformadores industriales revela el crecimiento de la demanda y la importancia económica del mercado de Dakar. La filial local, caracterizada por un crecimiento del número de pequeñas unidades de transformación, se desarrolla sobre todo en las regiones. Sin embargo, el dinamismo de este sector escapa a los políticos que deciden en Dakar, debido a la reclusión de las mini lecherías con respecto a los mercados de las ciudades secundarias en donde están implantadas. El Estado privilegia la cobertura de las necesidades de los consumidores de Dakar mediante las tarifas de aduanas, particularmente bajas para el polvo de leche transformado, que beneficia además de subvenciones en los países productores. Estas intervenciones en la filial local, basada sobre la intensificación (inseminación artificial), no han permitido el levantamiento de los múltiples obstáculos (salud animal, alimentación, agua, gestión de la calidad, colecta de la leche rural...). Parece entonces ser necesario que en la elaboración de las políticas lecheras se examine la filial de manera más global y favorecer una concertación con el conjunto de los actores de las dos sub filiales, para aumentar el impacto de las decisiones políticas, principalmente en términos de seguridad alimenticia y de lucha contra la pobreza en medio rural.

Palabras clave: Leche - Producto lácteo - Empresa pequeña Procesamiento - Diversificación - Senegal. 